\title{
Reversal of Chemotherapy Resistance to Cisplatin in NSCLC by miRNA-195-5p via Targeting the FGF2 Gene
}

\author{
Hao Wang ${ }^{1,2}$ \\ Zhi-lin Sui ${ }^{\prime}$ \\ Xian-xian $\mathrm{Wu}^{\prime}$ \\ Peng Tang' \\ Hong-dian Zhang' \\ Zhen-tao Yu ${ }^{1,3}$ \\ 'Department of Esophageal Cancer, \\ Tianjin Medical University Cancer \\ Institute and Hospital, Key Laboratory of \\ Cancer Prevention and Therapy of Tianjin \\ City, Tianjin, 300060, People's Republic of \\ China; ${ }^{2}$ Department of Surgical \\ Oncology, Baotou Cancer Hospital, \\ Baotou, People's Republic of China; \\ ${ }^{3}$ Department of Thoracic Surgery, \\ National Cancer Center, National \\ Clinical Research Center for Cancer, \\ Cancer Hospital \& Shenzhen Hospital, \\ Chinese Academy of Medical Sciences \\ and Peking Union Medical College, \\ Shenzhen, 518116, People's Republic of \\ China
}

Correspondence: Zhen-tao Yu; Hong-dian Zhang

Department of Esophageal Cancer, Tianjin Medical University Cancer Institute and Hospital, Key Laboratory of Cancer Prevention and Therapy of Tianjin City, Tianjin, 300060, People's Republic of China Tel +86 22-23340I23

Fax +86 22-23359984

Email yuzhentao@tjmuch.com;

zhdiantjzl@tmu.edu.cn
Objective: To explore the mechanism of miR-195-5p in the pathogenesis non-small cell lung cancer (NSCLC) and cisplatin resistance.

Methods: The function of miR-195-5p in NSCLC and cisplatin resistance were determined by MTT, scratch assay, transwell assay, and nude mice xenograft experiments. miR-195-5p target gene was identified by dual-luciferase reporter assays and real-time PCR analysis.

Results: miR-195-5p content was lower in A549/DDP than that in A549 cells, with reduced chemotherapy sensitivity and increased cell invasion and migration ability. The loss-offunction and gain-of-function assays illustrated that miR-195-5p might have increased the chemosensitivity to cisplatin in the A549/DDP cells and decreased cell migration and invasion. FGF2 is a negatively correlated action target of miR-195-5p. miR-195-5p might affect EMT by inhibiting FGF2. Overexpression of FGF2 resulted in enhanced cisplatin resistance in the cells, while miR-195-5p might have reversed this resistance.

Conclusion: Overall, miR-195-5p might target FGF2 to reduce cisplatin resistance in A549/ DDP cells and enhance chemosensitivity.

Keywords: miR-195-5p, NSCLC, cisplatin, chemosensitivity, FGF2

In 2018, there were 774,000 lung cancer (LC) cases and 691,000 deaths due to LC in China. ${ }^{1}$ Due to the lack of effective non-invasive screening and early treatment in most areas, the prognosis of LC is poor. Another primary reason for the poor prognosis of LC is chemotherapy resistance. Due to the distant metastasis and recurrence of lesions after local resection, surgical resection alone cannot effectively improve patients' survival. A combination of surgery and adjuvant and neoadjuvant chemotherapy is an effective means to improve the therapeutic effect. However, most patients will gradually develop drug and chemotherapy resistance in the later stage of treatment, resulting in the treatment's ultimate failure. ${ }^{2}$

MicroRNAs (miRNAs) are endogenous non-protein-coding RNAs with a length of 21-25 nt in eukaryotes that regulate more than $60 \%$ of protein-coding genes, influence physiological responses to development, growth, aging, and disease, and play an important role in tumor resistance to chemotherapy by regulating apoptosis, the cell cycle, drug transport, and drug resistance gene expression. ${ }^{3}$

Specifically, miR-195-5p shows low expression in many malignant tumors, eg, LC, prostate cancer, liver cancer, gastric cancer, ovarian cancer, and cervical cancer, and is closely correlated with tumorigenesis, progression, metastasis, and prognosis. 
It also has an impact on the sensitivity of chemotherapeutic agents. ${ }^{4}$ However, the role of miR-195 in chemotherapy resistance in LC has not been heavily studied.

Fibroblast growth factor 2 (FGF2) is one of the 23 members of the FGF family. In addition to participating in many pathophysiological processes, including angiogenesis and tissue repair, FGF2 also regulates immune cell activity, influences the function of the immune system, and is very important to inhibit tumor resistance to chemotherapy. ${ }^{5}$ We focused on the effect of miR-195 in the chemotherapy resistance of non-small cell LC (NSCLC) and its relationship with the target gene FGF2, aiming to provide new ideas for NSCLC treatment.

\section{Methods}

\section{Cell Culture}

The human NSCLC cell line A549 and cisplatin (DDP)resistant cell line A549/DDP were purchased from Tianjin Saier Biotechnology Co., China, and cultured in RPMI1640 solution. To maintain the cells' normal phenotypic characteristics, the cell culture medium contained $10 \%$ fetal bovine serum (FBS), streptomycin $(100 \mathrm{~mol} / \mathrm{mL})$, and penicillin $(100 \mathrm{u} / \mathrm{mL})$, and the culture was placed in a constant-temperature incubator at $37^{\circ} \mathrm{C}$ and $5 \% \mathrm{CO}_{2}$. Cells were routinely cultured for one week in the medium without DDP.

\section{Plasmid Construction and Cell Transfection}

miRNA mimic oligonucleotide duplexes and small interfering RNA (siRNA) were chemically synthesised by GenePharma (Shanghai, China).Cells were seeded in sixwell plates and cultured until $50 \%$ to $70 \%$ confluency. Then cells were transfected by Lipofectamine 2000 (Invitrogen, Carlsbad, New Mexico, USA). The transfection mixture was replaced with a medium containing $10 \%$ foetal bovine serum (FBS) after $6 \mathrm{~h}$ to $8 \mathrm{~h}$.

The commercially synthesized miR-195-5p-mimics and miR-195-5p-ASO were purchased from GenePharma (Shanghai, China). The pSilencer/shR-FGF2 (shR-FGF2) plasmids expressing siRNA targeting FGF2 were constructed by annealing double-strand hairpin cDNA and inserting it into the pSilencer 2.1-U6 neo vector (Ambion, Austin, TX, USA). The relevant control vector is pSilencer 2.1-U6 neo, which was named "pSilencer$\mathrm{NC}$ " in short in paper. The full-length sequences of human FGF2 cDNA (NM_002006) were obtained by
RT-PCR and cloned into pcDNA3 at HindIII and KpnI sites. The resulting plasmid was termed pcDNA3/FGF2. All primers for PCR amplification are provided in Table 1. Transient transfection was performed in antibiotic-free Opti-MEM medium (Invitrogen) with the Lipofectamine 2000 reagent (Invitrogen, Carlsbad, CA) following the manufacturer's protocol. The transfection mixture was replaced with a medium containing $10 \%$ foetal bovine serum (FBS) after $6 \mathrm{~h}$. After $48 \mathrm{~h}$ after transfection, cells were collected for Western blot (WB) analysis and reverse transcription polymerase chain reaction.

\section{RNA Isolation and RT-qPCR}

The total RNAs was extracted from the A549 and A549/ DDP cells using the miR-Vana miRNA Isolation Kit (Ambion, Austin, TX, USA). Complementary DNA (cDNA) was obtained by reverse transcription of total cellular RNA using M-MLV reverse transcriptase (Promega, Madison, WI, USA), After the reverse transcription, the expression of miR-195-5p and FGF2 were detected by RT-qPCR using an iQ5 Real-Time PCR Amplifier with U6 as the internal reference. All the primers (Table 1) were purchased from AuGCT, Inc. (Beijing, China).

\section{Western Blotting Analysis}

After washing the A549 and A549/DDP cells with precooled phosphate-buffered saline (PBS) three times, each group of cells was lysed on ice with RIPA buffer (Saier Biotechnology Co. Tianjin, China) for $30 \mathrm{~min}$. The cell protein sample was obtained by transferring the cell lysate to a $1.5 \mathrm{~mL}$ centrifuge tube. Then, the protein sample went SDS denaturing $10 \%$ polyacrylamide gel electrophoresis (SDS-PAGE) until the target proteins were effectively separated. The proteins were electrotransferred to a PVDF membrane, and blocked for $2 \mathrm{~h}$ with $5 \%$ skim milk at room temperature. After the proteins were incubated with primary antibodies at $4^{\circ} \mathrm{C}$ overnight, the blots were washed four times with PBS, incubated with horseradish peroxidase (HRP)labelled secondary antibody at room temperature for 2 $h$ and visualised through enhanced chemiluminescence (Saier Biotechnology Co. Tianjin, China). The grayscale values of the protein bands were analyzed using the Image $J$, and the expression of each target protein was calculated with GAPDH or $\beta$-actin used as the internal reference. The following primary antibodies 
Table I The Primers and Oligonucleotides Used in This Work

\begin{tabular}{|c|c|}
\hline Name & Sequence $\left(5^{\prime}-3^{\prime}\right)$ \\
\hline has-miR-195-5p-mimics & UAGCAGCACAGAAAUAUUGGC \\
\hline has-miR-195-ASO & GCCAAUAUUUCUGUGCUGCUA \\
\hline has-miR-195-RT & GTCGTATCCAGTGCAGGGTCCGAGGTGCACTGGATACGACGCCAATAT \\
\hline has-miR-195-Fwd & TGCGGTAGCAGCACAGAAATATTGGC \\
\hline U6 RT & GTCGTATCCAGTGCAGGGTCCGAGGTATTCGCACTGGATACGACAAAATATGGAAC \\
\hline U6 Fwd & TGCGGGTGCTCGCTTCGGCAGC \\
\hline Reverse & CCAGTGCAGGGTCCGAGGT \\
\hline PmiRGLO-FGF2-3'UTR-top & AAACTAGCGGCCGCTAGTTAATTTAAAATATTTTGCTGCTAGTTAACTATGT \\
\hline PmiRGLO-FGF2-3'UTR-bot & CTAGACATAGTTAACTAGCAGCAAAATATTTTAAATTAACTAGCGGCCGCTAGTTT \\
\hline PmiRGLO-FGF2-3'UTR-mut-top & AAACTAGCGGCCGCTAGTTAATTTAAAATATTTTACACGAAGTTAACTATGT \\
\hline PmiRGLO-FGF2-3'UTR-mut-bot & CTAGACATAGTTAACTTCGTGTAAAATATTTTAAATTAACTAGCGGCCGCTAGTTT \\
\hline shR-FGF2-top & GATCCTATAGCTCAGTTTGGATAATTCTCGAGAATTATCCAAACTGAGCTATATTTTTGA \\
\hline shR-FGF2-bot & AGCTTCAAAAATATAGCTCAGTTTGGATAATTCTCGAGAATTATCCAAACTGAGCTATAG \\
\hline pcDNA3-FGF2 -S & TATAAGCTTCT GGTGGGTGTG GGGGGTGGAGAT \\
\hline PcDNA3-FGF2-AS & CGGGGTACCGCTCTTAGCAGACATTGGAAG \\
\hline FGF2-qPCR-Fwd & AAGAGCGACCCTCACATCAAG \\
\hline FGF2-qPCR-Rev & CGTTTCAGTGCCACATACCAAC \\
\hline$\beta$-actin-qPCR-Fwd & CGTGACATTAAGGAGAAGCTG \\
\hline$\beta$-actin-qPCR-Rev & CTAGAAGCATTTGCGGTGGAC \\
\hline
\end{tabular}

were used in the Western blot assay: (1) rabbit antiGAPDH (Tianjin Saier Biotech; 1:1000), (2) rabbit anti-E-cadherin (Tianjin Saier Biotech; 1:1000), (3) rabbit anti-vimentin (Tianjin Saier Biotech; 1:1000), (4) rabbit anti-FGF2 (Tianjin Saier Biotech; 1:1000).

\section{Luciferase Assay}

Bioinformatic analysis software TargetScanHuman 7.2, target gene prediction software, and miRanda were applied to perform the bioinformatic functional analysis of miR195-5p and predict target genes (https://www.targetscan. org). Luciferase reporter assays were co-transfected in 48well plates with $0.5 \mu \mathrm{g}$ of mimics-NC or miR-195-5p mimics and $0.5 \mu \mathrm{g}$ of reporter vector FGF2-3'UTR or FGF2-3'UTR mut. The activities of the firefly luciferase and the Renilla luciferase were detected using the DualGlo system (Promega, Madison, WI) 48 h posttransfection.

\section{The Cell Function Tests}

\section{Methyl Thiazole Tetrazole (MTT) Assay}

The cells, following transfection, were seeded at a density of 3,000 cells per well, and cell activity was detected by MTT at $48 \mathrm{~h}$ after transfection (incubated with $10 \mu \mathrm{L}$ MTT solution at a concentration of $10 \mathrm{mg} / \mathrm{mL}$ at $37^{\circ} \mathrm{C}$ for $4 \mathrm{~h}$ ). The original culture solution was then removed, and added $100 \mu \mathrm{L}$ DMSO to each well. After shaking for $10 \mathrm{~min}$, on a shaker to fully dissolve the methylzan the blue-purple crystalline material. The absorbance values of each well were measured at $570 \mathrm{~nm}$ via an uQuant Universal Microplate Spectrophotometer (Bio-Tek Instruments, Winooski. USA).

\section{The Scratch Healing Assay}

Transfected cells were scraped and at least three artificial wounds were photographed using 10 high-power fields at $0 \mathrm{~h}, 24 \mathrm{~h}$ and $48 \mathrm{~h}$ after scratch. 


\section{The Invasion Assay}

For the invasion assay, $6 \times 10^{4}$ A549 cells or A549/DDP cells were seeded on upper chamber of the inserts coated by $40 \mu \mathrm{L}$ Matrigel (DB Biosciences) diluted to $2 \mu \mathrm{g} / \mu \mathrm{L}$. Meanwhile, the bottom of the inserts were incubated in medium containing $20 \%$ FBS. After cultured for $48 \mathrm{~h}$, the number of cells migration or invasion to the bottom of chamber was counted per field.

\section{Mouse Model of Tumor Xenograft}

After transfection, $1 \times 10^{7}$ A549 and A549/DDP cells were injected into the endermic of 6-week-female BALB/c nude mice (4 per group). Tumor size was measured every four days after two weeks of injection. The tumor volume was calculated as follows: length $\times$ width ${ }^{2} \times 1 / 2$. All mice were sacrificed 5 weeks after implantation, the tumors were isolated from the mice and stored at $-80^{\circ} \mathrm{C}$. Which is approved by Animal Use Committee of Tianjin Medical University Cancer Institute and Hospital (Approved No.: NSFC-AE2020103) and complied with the National Institutes of Health Guide for the Care and Use of Laboratory Animals.

\section{Statistical Analysis}

All of the experiments were repeated three times. The SPSS 21.0 and GraphPad Prism7 software were adopted for the statistical analysis. The measurement data conformed to the normal distribution were expressed as $\bar{X} \pm$ $\mathrm{s}$, and $t$-test was used to compare means between two groups. For the comparison of means among multiple groups, the homogeneity of variance was tested first, and then one-way ANOVA was conducted; $\mathrm{P}<0.05$ was considered statistically significant.

\section{Results}

\section{Biological Efficacy in A549/DDP and A549 Cells}

To explore the differences between A549/DDP and A549 cells. The results showed that the $\mathrm{IC}_{50}$ of DDP on the A549 cells was $0.8 \mu \mathrm{g} / \mathrm{mL}$, while the $\mathrm{IC}_{50}$ of DDP on the A549/DDP cells was $2.2 \mu \mathrm{g} / \mathrm{mL}$, These results showed that the inhibitory effect of DDP on the activity of A549 cells was stronger than that of A549/DDP cells and A549/ DDP cells were less sensitive to DDP than A549 cells. (Figure 1A). The scratch assay revealed that the migration rate of the A549 cells after $24 \mathrm{~h}$ was $20 \%$, significantly lower than the migration rate of $57 \%$ in the A549/DDP cells. This suggested that the A549 cells had a lower cell migration ability compared to the A549/DDP cells (Figure 1B). Moreover, the invasion ability of the A549 cells was weaker than that of the A549/DDP cells (Figure 1C).

\section{The Effects of miR-195-5p on the Cell Function}

The expression of miR-195-5p in the A549/DDP cells was lower than that in the A549 cells (Figure 2A). MTT assay showed increased sensitivity and decreased the viability of A549/DDP cells after transfection with miR-195-5p mimics via the action of cisplatin. Meanwhile, the A549 cells transfected with the miR195-5p-ASO had a reduced sensitivity, increased cells viability, and increased cisplatin resistance (Figure 2B and C). The migration and invasion ability of the A549/ DDP cells decreased after transfection with miR-195-5p mimics, while the migration and invasion ability of the A549 cells transfected with the miR-195-5p-ASO increased significantly (Figure 2D and E).

We then examined whether the molecular changes typical to affected EMT occurred in A549 and A549/ DDP cells after transfected miR-195-5p or miR-195-5pASO. miR-195-5p increased the expression levels of E-cadherin, but decreased the level of vimentin, while the opposite results were observed in A549 cells after transfected miR-195-5p-ASO (Figure 2F). These results suggested that miR-195-5p inhibits the epithelial-like/ mesenchymal-like phenotype.

\section{Prediction and Verification of Target Genes of miR-195-5p}

The bioinformatics functional analysis showed that the mature sequence of miR-195-5p is "UAGCAGCACAG AAAUAUUGGC,, which is highly conserved among species. Target prediction using bioinformatics methods suggested the candidate targets for miR-195-5p, we chose FGF2 as putative target for further study after considering available functional knowledge. The luciferase activities were decreased when FGF2 $3^{\prime}$ UTR and miR-195-5p were co-transfected, whereas co-transfection with miR-195-5p-ASO caused a increase compared with the control (Figure 3A). In contrast, mutation of the miR-195-5p binding site in the FGF2 3' UTR abolished the effect of both miR-195-5p over-expression and miR-195-5p-ASO on the fluorescence intensity of the 


\section{A}
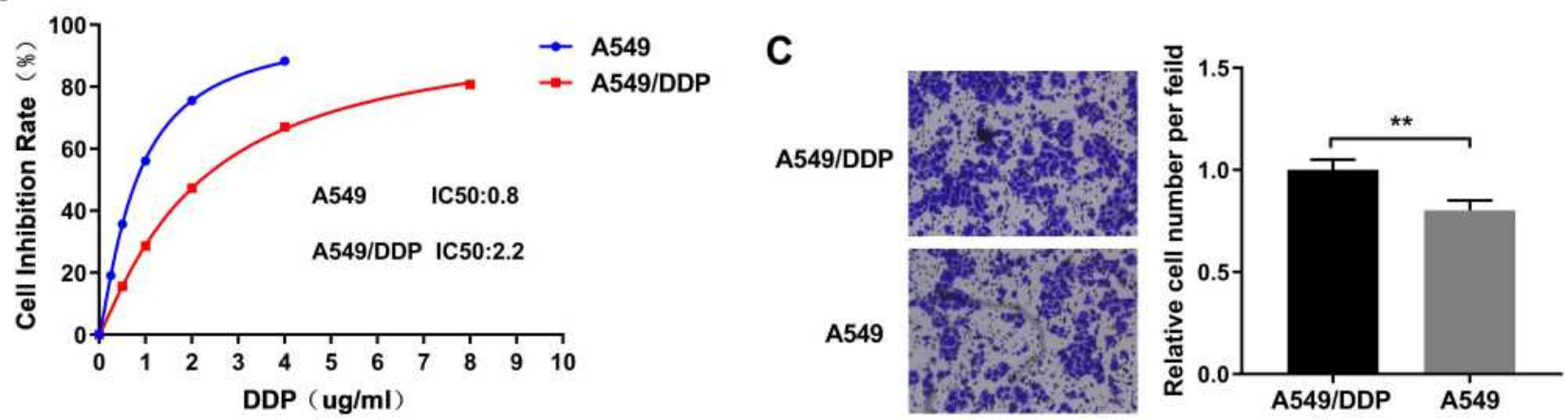

B
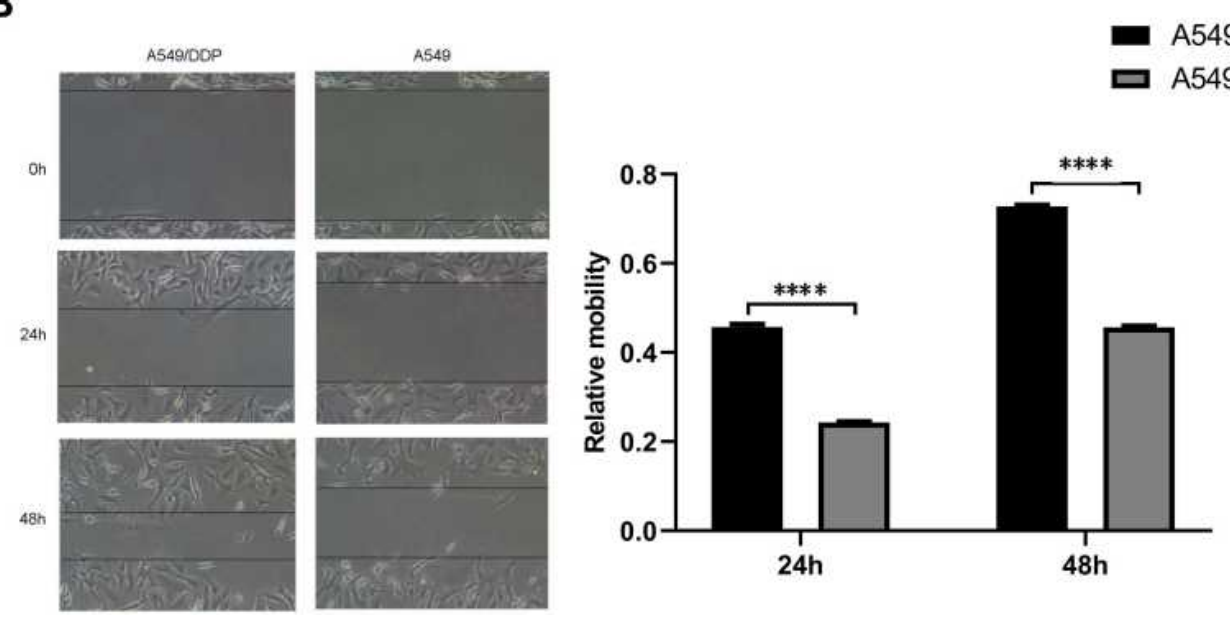

Figure I The difference in biological efficacy A549 and A549/DDP cells. (A) The MTT assay was used to detect the difference in cisplatin chemosensitivity of the two cell lines. The cisplatin sensitivity of A549/DDP by the MTT assay, IC50 $=2.2 \mu \mathrm{g} / \mathrm{mL}$. The cisplatin sensitivity of A549 by the MTT assay, IC50 $=0.8 \mu \mathrm{g} / \mathrm{mL}(\mathbf{B}) \mathrm{Wound}$ healing assays were performed to reveal the relative mobility. The line graphs show the mean \pm SD of three scratches from one representative experiment. (C) $24 \mathrm{~h}$ after transfection, A549 and A549/DDP cells (in $200 \mu \mathrm{L}$ serum-free medium) were seeded into inserts and incubated for $60 \mathrm{~h}$, chambers were disassembled, and the membranes were stained with $2 \%$ crystal violet solution for 5 min and placed on a glass slide. Cells at the lower surface of the membrane were counted under a light microscope at five random fields. All data represent mean \pm SD of three independent experiments. ${ }^{* *} \mathrm{p}<0.01$. The invasion ability of the A549 cells was weaker than that of the A549/DDP cells. All data represent mean \pm SD of three independent experiments. ${ }^{* * * *} \mathrm{p}<0.0001$.

luciferase activities (Figure 3B). These results suggest that miR-195-5p may directly target FGF2.Next we further determined the effect of miR-195-5p on endogenous FGF2 expression. RT-qPCR showed that FGF2 mRNA levels were decreased in A549 and A549/DDP cells transfected with miR-195-5p (Figure 3C and D). Furthermore, Western blotting assays showed that miR195-5p decreased FGF2 expression (Figure 3E and F). These results indicate that miR-195-5p suppresses FGF2 expression at both the mRNA and protein levels in both cells. Furthermore, protein and mRNA expression results of FGF2 in the A549 and A549/DDP cells detected by WB and RT-qPCR demonstrated a significant difference in the expressions between the A549 and A549/DDP cells (Figure 3G).

\section{Effect of MiR-195-5p on the Sensitivity of A549 and A549/DDP Cells to Cisplatin}

Sensitivity to cisplatin in the different groups was measured as the $\mathrm{IC}_{50}$ concentration after transfection. Growth inhibition rate of the A549 cells was $52.0 \%$ under the effect of cisplatin at an $\mathrm{IC}_{50}$ concentration of $0.8 \mu \mathrm{g} / \mathrm{mL}$ but decreased to $37.6 \%$ after transfection with miR-195$5 \mathrm{p}$-ASO. Moreover, inhibition rate of cell growth of A549 cells increased to $75.5 \%$ after transfection with shR-FGF2 alone, while the growth inhibition rate of the A549 cells after transfection of miR-195-5p-ASO and shR-FGF2 was 46.7\% (Figure 4A). In the A549/DDP cells, growth inhibition rate after miR-195-5p transfection increased from $50.3 \%$ to $66.0 \%$ under the effect of cisplatin at an $\mathrm{IC}_{50}$ concentration of $2.2 \mu \mathrm{g} / \mathrm{mL}$. The growth inhibition rate of 

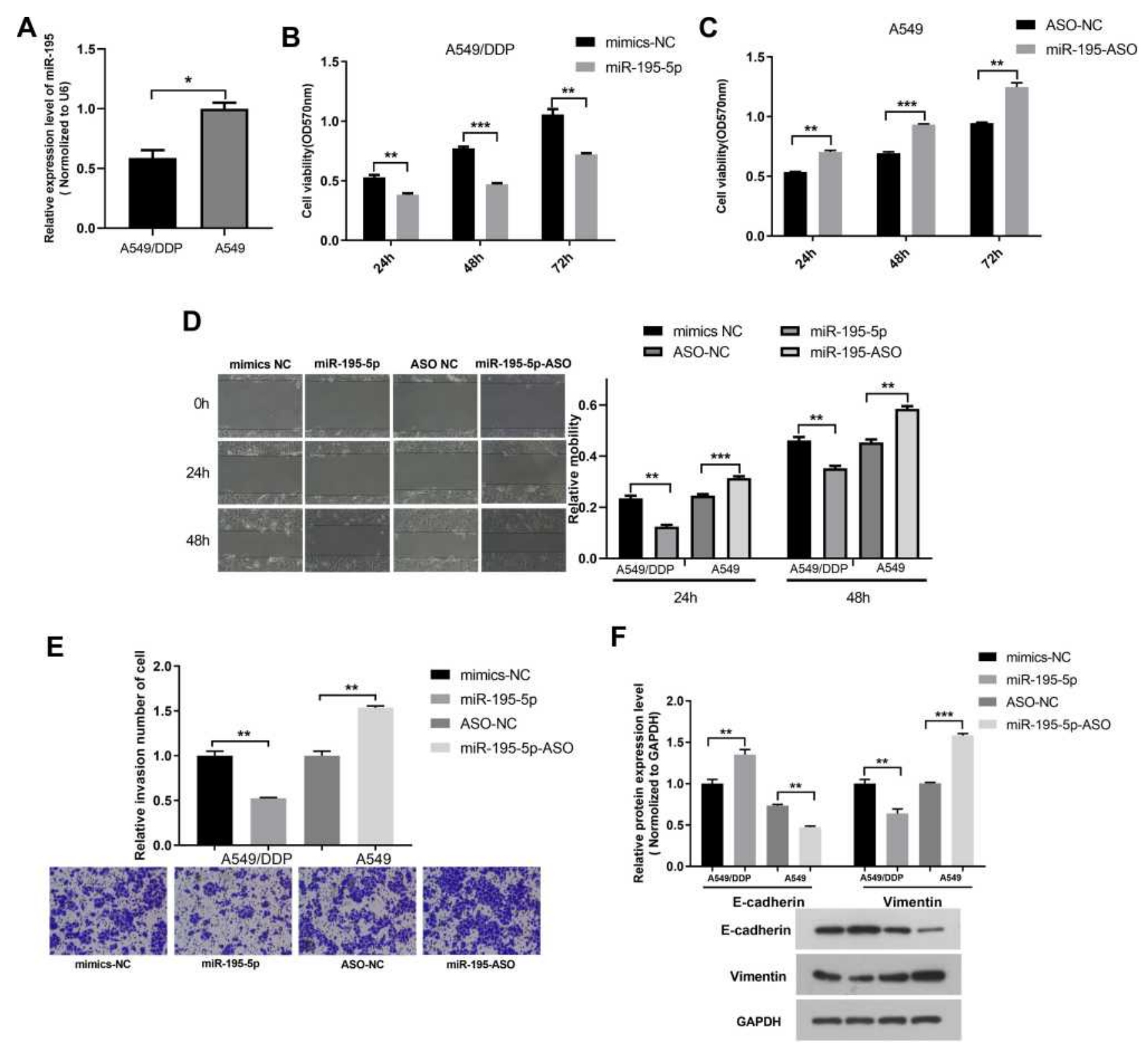

Figure 2 The effects of miR-195-5p on the cell function of the A549 and A549/DDP cells. (A) The miR-195-5p expression was examined in A549 and A549/DDP cells using RT-qPCR. (B and C) MTT assay was applied to determine the cell viability of A549/DDP and A549 cells. (D) Wound healing assays were measured in A549 and A549/DDP cells with miR-195-5p mimics or inhibitor. (E) Cell invasion analysis in cells with miR-195-5p mimics or inhibitor were detected. (F) Protein expression levels of EMTassociated markers were assessed by Western blotting and quantification was presented in bar graphs. All data represent mean \pm SD of three independent experiments. ${ }^{*} \mathrm{P}<$ $0.05, * * p<0.01, * * * p<0.001$.

the A549/DDP cells after transfection with pcDNA3-FGF2 alone was $31.1 \%$, while the growth inhibition rate of the A549/DDP cells after simultaneous transfection with the miR-195-5p and pcDNA3-FGF2 was 46.7\% (Figure 4B).

\section{Over-Expression of FGF2 Abolishes the Effects Induced by miR-195-5p in A549/ DDP Cells}

The effects of miR-195-5p overexpression on mobility, migration and invasion were significantly impaired when
FGF2 was overexpressed (Figure 5A-C). Furthermore, the expression of EMT markers in the miR-195-5pexpressing cells were restored to the normal levels after overexpression of FGF2 (Figure 5D). Conversely, knockdown of FGF2 expression in A549 cells abolished the promotion effect of miR-195-ASO on cell activity, migration, invasion, and EMT (Figure 5E-H). Collectively, these results indicate that the effects of miR-195-5p are probably due to the knockdown of FGF2. 


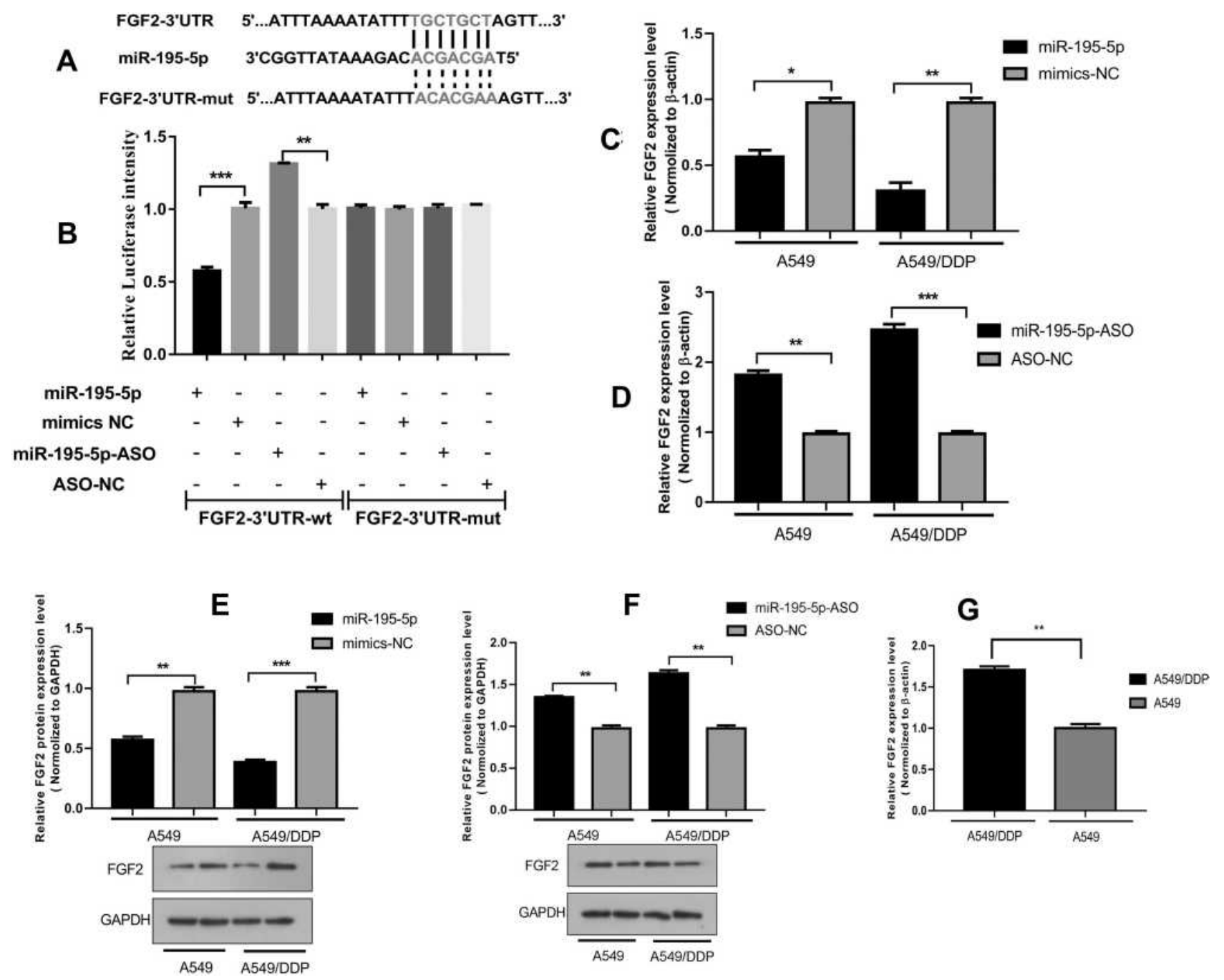

Figure 3 The miR-195-5p target gene prediction and verification. (A) The predicted miR-195-5p-binding sites in FGF2 mRNA using Targetscan 7.0 are shown. (B) A549 cells were transfected with PmiRGLO reporter vectors containing either wild-type or mutant FGF2 3 'UTR. The relative Luciferase activity was determined after transfection. (C and D) The expression of FGF2 was analyzed in cells with miR-195-5p mimics or inhibitor. (E and $\mathbf{F}$ ) The expression of FGF2 protein was detected by WB in cells with miR-195-5p mimics or inhibitor. (G) The difference expression of FGF2 was analyzed by RT-qPCR in A549 and A549/DDP cells. All data represent mean \pm $\mathrm{SD}$ of three independent experiments. ${ }^{*} \mathrm{p}<0.05,{ }^{*} \mathrm{p}<0.01$, ${ }^{* * *} \mathrm{p}<0.001$.

\section{miR-195-5p Overexpression Inhibit NSCLC}

Tumor xenograft experiments was performed using A549/ DDP cells transfected with miR-195-5p and A549 cells transfected with miR-195-5p-ASO. miR-195-5p overexpression inhibited and knockdown miR-195-5p promoted growth of NSCLC in vivo (Figure 6A-C). At the same time, tumor in the group injected with A549/DDP cells were larger than those injected with A549 cells. RTqPCR and Western blot analysis showed that the protein and mRNA expressions of FGF2 in the tumor-forming tissues of the A549/DDP cells were significantly higher than those of FGF2 in the tumor-forming tissues of the A549 cells. Meanwhile, the miR-195-5p expression in the tumor tissues of the A549/DDP cells was significantly lower than that in the tumor-forming tissues of the A549 cells. (Figure 6D-F).

\section{Discussion}

According to statistics by the National Cancer Institute of the USA, five-year overall survival was $23.6 \%$ in NSCLC ${ }^{6}$ with rates of approximately $55.6 \%$ for NSCLC without metastasis and $28.9 \%$ for NSCLC with regional lymph node metastasis. Once distant metastasis occurred, the five-year survival was only $4.5 \%$.

One of the main reasons is drug resistance to chemotherapy, which is a complex process with many influencing factors, primarily individual genetic differences, the 

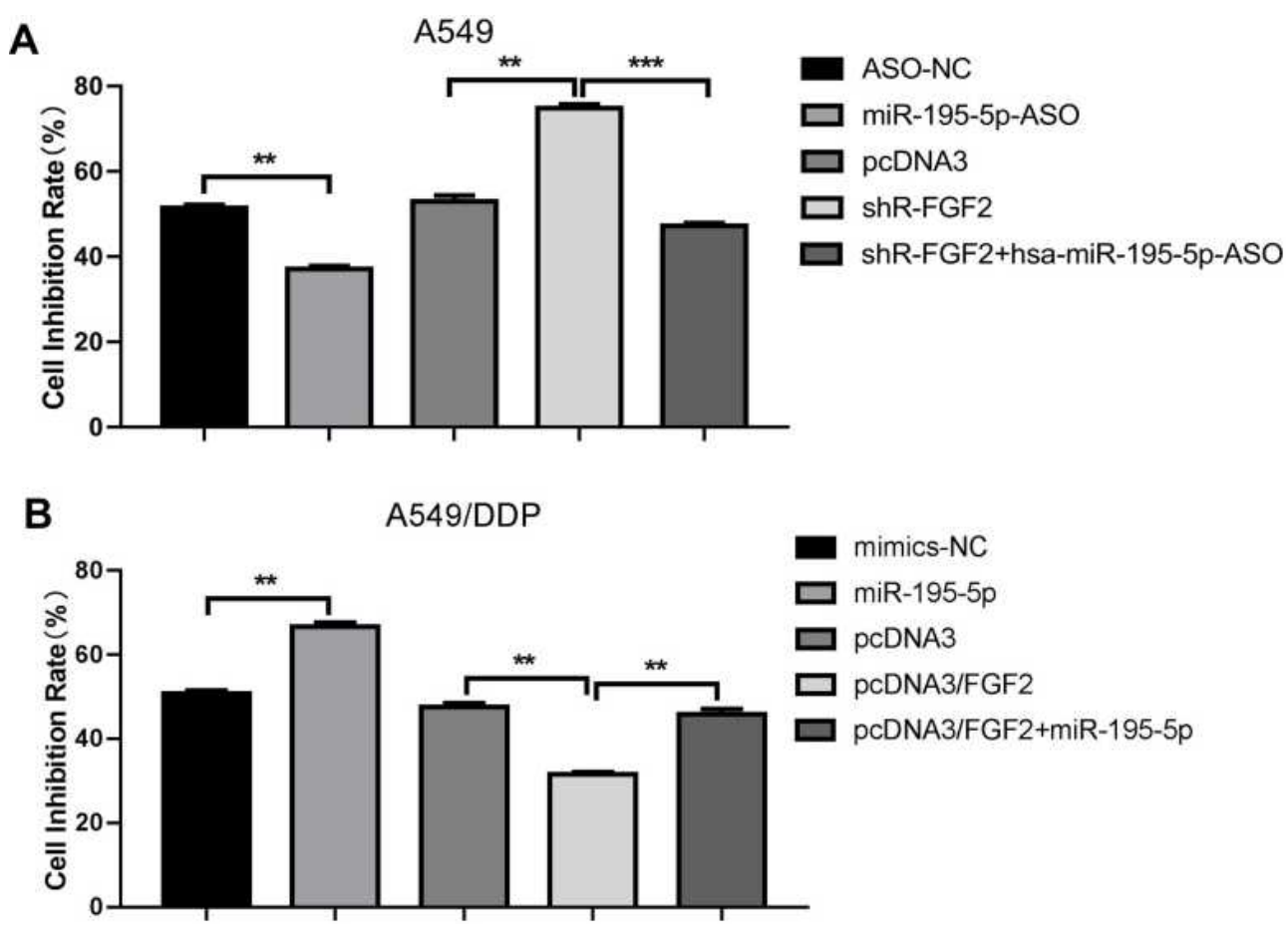

Figure 4 MiR-195-5p increased the sensitivity of A549 and A549/DDP cells to cisplatin by inhibiting the target gene FGF2. (A and B) An MTT assay tested the cells sensitivity of A549 and A549/DDP cells to cisplatin. All data represent mean \pm SD of three independent experiments. $* * p<0.01$, $* * * p<0.001$.

tumor microenvironment, tumor stem cells, drug inactivation, reduced drug absorption, and altered metabolism of antitumor drugs. Some miRNAs can act on drug efflux pump molecules, apoptosis regulatory proteins, and cycle regulatory proteins to alter the drug-resistance of tumor cells. $^{7}$ Cisplatin is an early developed and clinically mature drug for the treatment of LC and has remained the drug of choice for LC chemotherapy up to now. However, chemotherapy resistance limits the efficacy of cisplatin in clinical use. After entering the body, cisplatin dissociates in a low-chloride environment to form hydrated cations, which mainly act on DNA, forming covalent bonds with DNA and, thus, limiting DNA deconvolution and impeding DNA replication to inhibit the division of cancer cells and promote their apoptosis and necrosis. $^{8}$

The miR-195 gene is a newly found micro RNA of the miR-15/107 family, which is homologous to miRNA-15, miRNA-16, miRNA-424, and miRNA-497 and shares the 5 'core sequence of CGA CGA. ${ }^{9}$ The dysregulation of miR195 will cause the development of many diseases; miRNA-195 was first found to be highly expressed in transgenic mice with cardiac hypertrophy and to cause progression from the pathological heart disease to heart failure. $^{10}$
The high expression of miR-195 can inhibit tumorigenesis and progression and affect the sensitivity of chemotherapeutic agents for malignant tumors, a property that has been applied to drug development. Therefore, it was considered that propofol might induce miR-195 to inhibit the proliferation, migration, and invasion of gastric cancer cells. ${ }^{11}$ Zuo et al investigated miR-195 together with long-chain non-coding 00485 (LINC00485) in cisplatin resistance and found that overexpression of miR-195 or silencing of LINC00485 enhances the sensitivity of lung adenocarcinoma cells to cisplatin. ${ }^{12}$ These studies provided a theoretical basis for drug resistance studies in LC chemotherapy.

We found that the miR-195-5p expression in NSCLC resistant cells was significantly lower than that in the parental strain cells, while the chemotherapy sensitivity was decreased and the cell migration and invasion functions increased. Subsequently, we demonstrated that overexpression of miR-195-5p improved the chemosensitivity of cisplatin-resistant cells and reduced their migration and invasion functions. We found that miR-195-5p might play a role as an oncogene in LC cells and have an inhibitory effect on chemotherapy resistance to cisplatin. Afterward, by target gene prediction and confirmation, it was concluded that miR-195-5p might directly target downstream 
A

$\begin{aligned} & =p c D N A 3 \\ & =p c D N A 3 / F G F 2 \\ & =p c D N A 3 / F G F 2+\text { mimics-NC } \\ & p c D N A 3 / F G F 2+m i R-195-5 p\end{aligned}$

A549

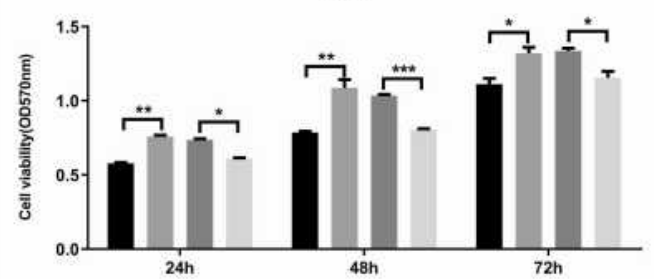

C

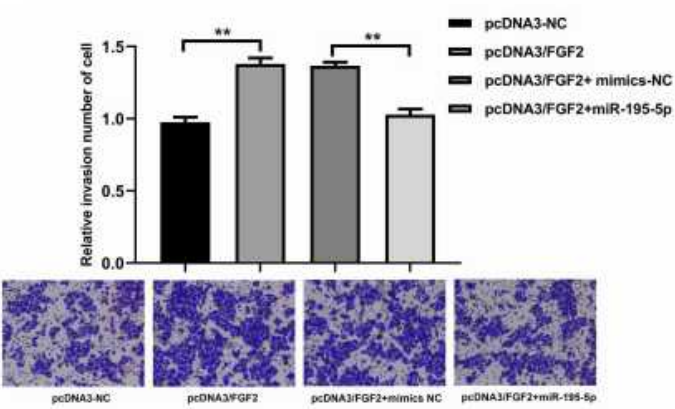

E

$$
\begin{aligned}
& \text { pSilencer-NC } \\
& \text { EhR-FGF2 } \\
& \text { = } \\
& \text { ahR-FGF2+ASO-NC } \\
& \text { shR-FGF2+miR-195-ASO }
\end{aligned}
$$

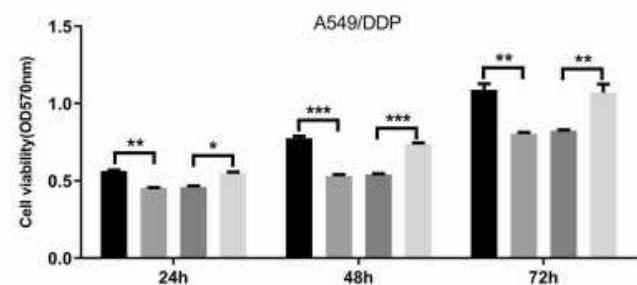

G

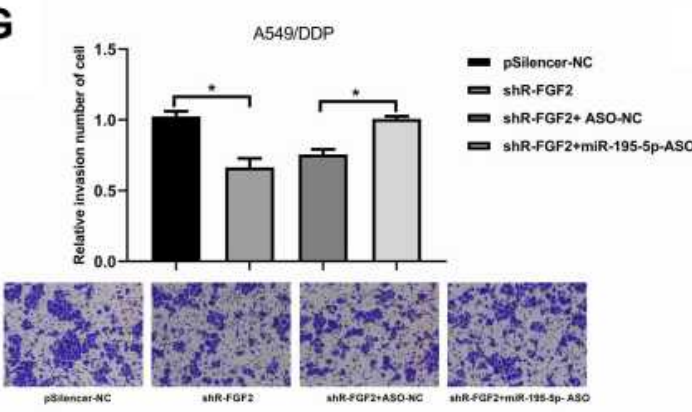

F

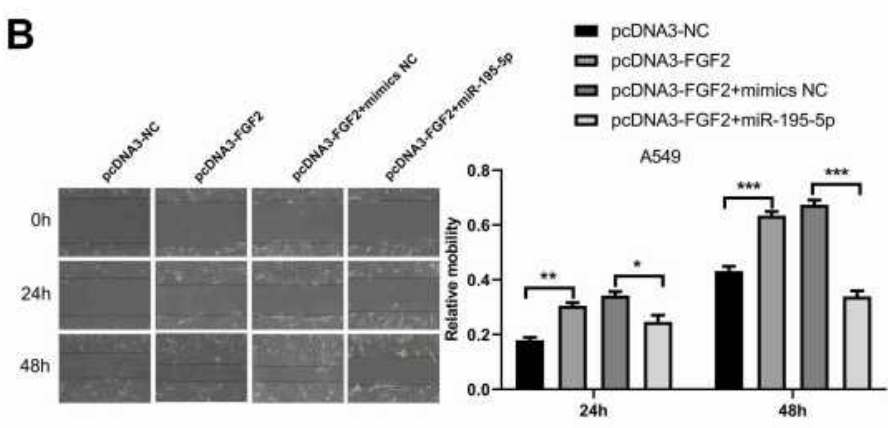

D
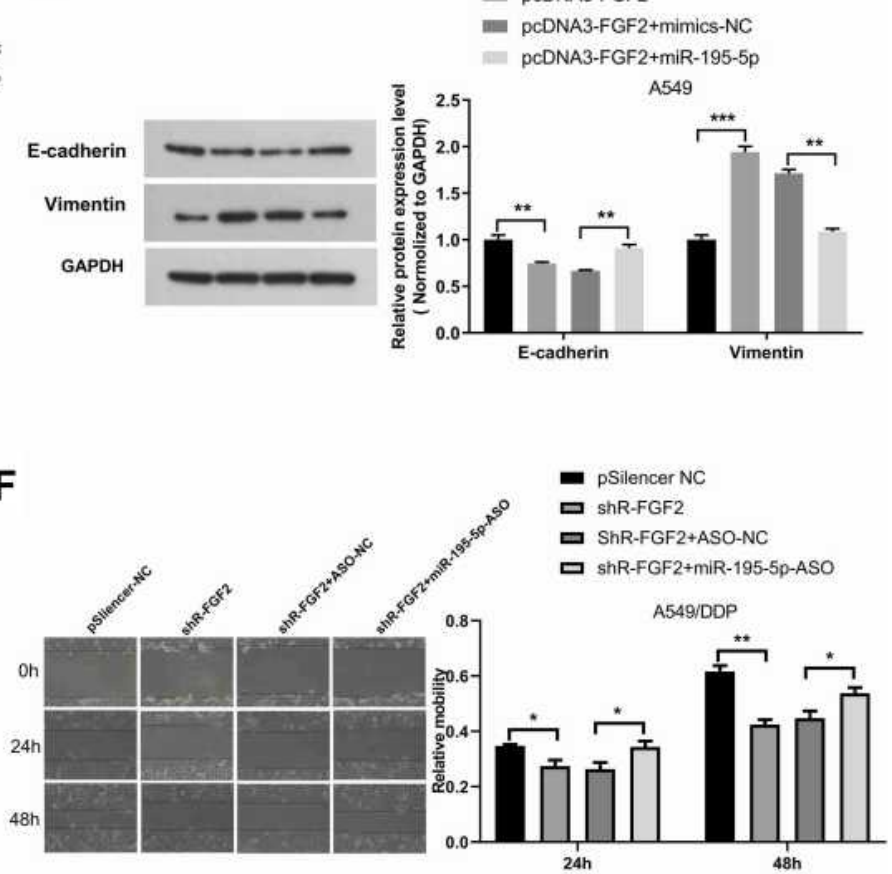

H

- pSilencer-NC

shR-FGF2

= ShR-FGF2+ASO-NC

= shR-FGF2+miR-195-5p-ASO
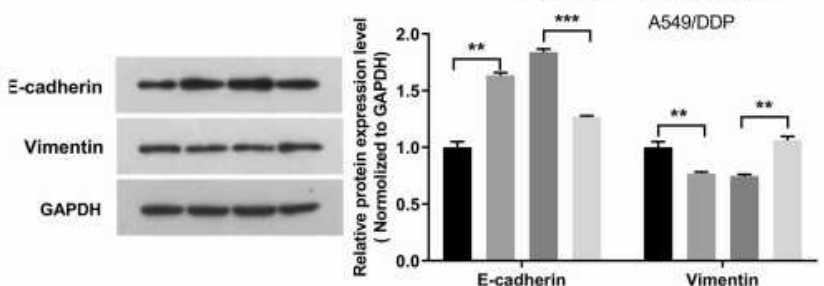

Figure 5 Over-expression of FGF2 abolishes the effects induced by miR-195-5p in A549/DDP cells. (A-C) Over-expression of FGF2 rescues the effect of miR-195-5p on cell viability (A), migration (B) and invasion (C) in A549/DDP cells. (D) After co-transfection with miR-195-5p and FGF2 in A549/DDP, Western blot assays were used to present the expression of EMT markers (E-cadherin and vimentin). (E-G) Knockdown of FGF2 rescues the effect of miR-195-5p on cell viability (E), migration (F) and invasion (G) in A549 cells. (H) After co-transfection with miR-195-5p-ASO and shR-FGF2 in A549, Western blot assays were used to present the expression of EMT markers (E-cadherin and vimentin). All data represent mean $\pm \mathrm{SD}$ of three independent experiments. ${ }^{*} \mathrm{p}<0.05$, ${ }^{* *} \mathrm{p}<0.0 \mathrm{I}, * * * \mathrm{p}<0.00 \mathrm{I}$. 
A

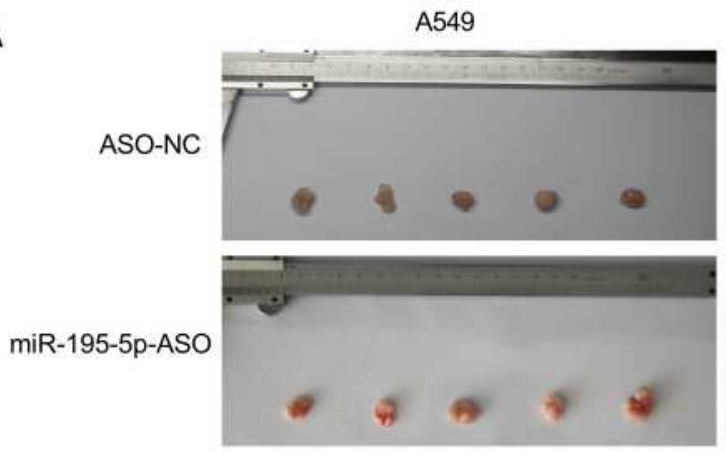

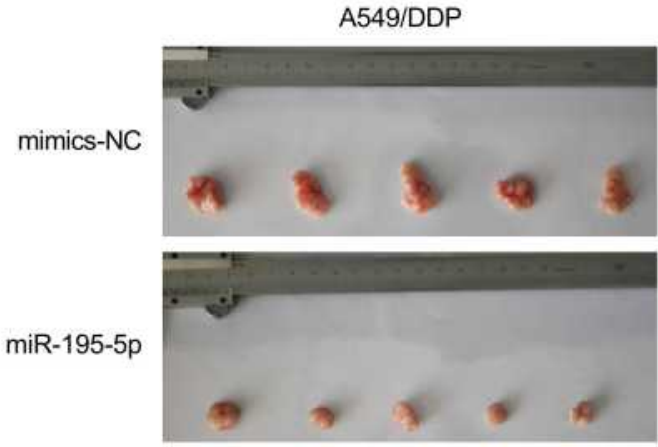

C
B

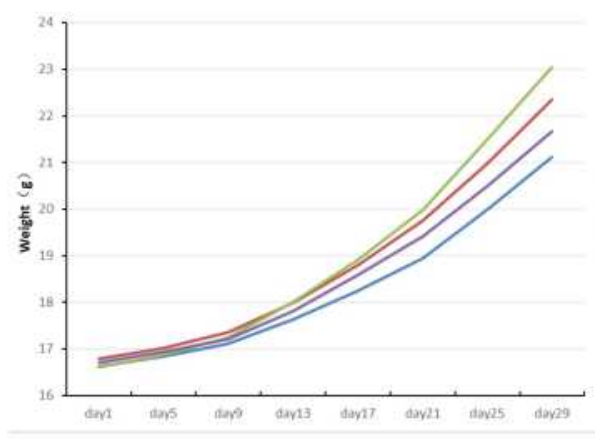

D

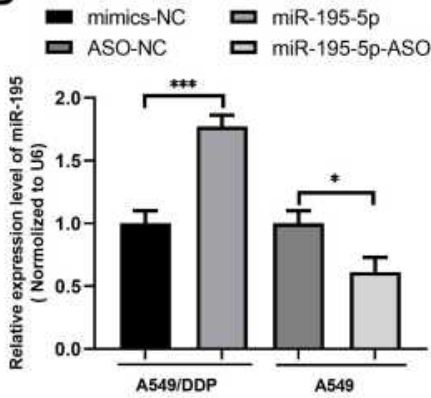

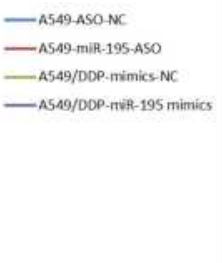

E

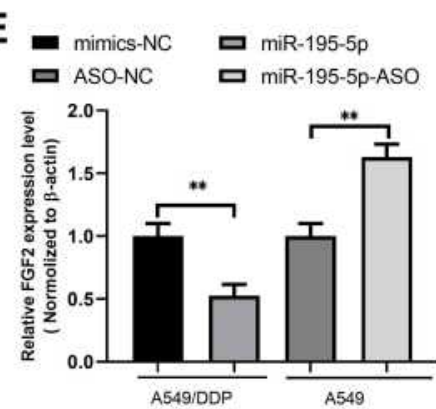

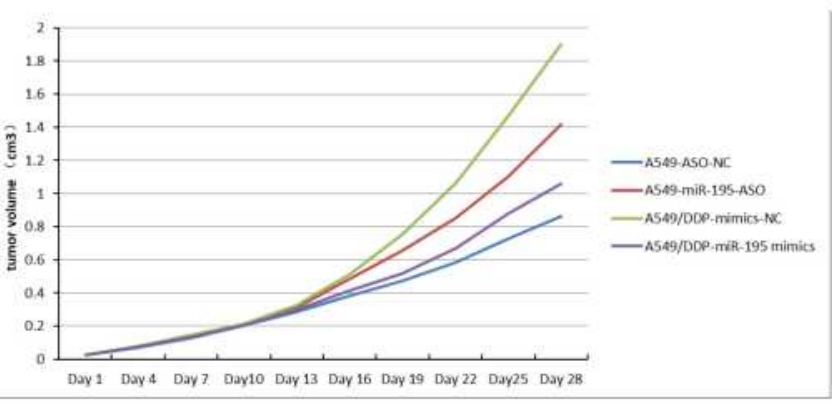

F

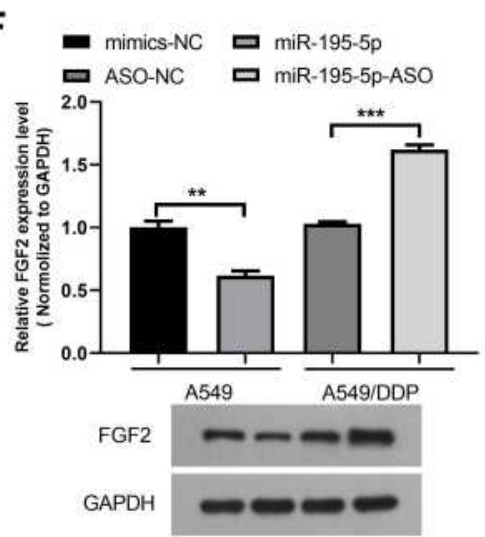

Figure 6 miR-195-5p over-expression inhibit NSCLC in vivo. (A) The mice were euthanized, and the tumors were isolated 5 weeks after implantation. (B)The body weight growth curve of nude mice was studied after cell injection. (C) Tumor growth curves of studied nude mice after cell injection was showed. (D and E) miR-I95-5p expression level and FGF2 mRNA expression level of nodules in mice. (F) FGF2 protein expression level of nodules in mice. All data represent mean \pm SD of three independent experiments. ${ }^{*} p<0.05$, $*_{p}<0.01$, ${ }^{*} * *_{p}<0.001$.

gene FGF2 to affect the resistance to cisplatin and migration invasion ability of LC cells.

Fibroblast growth factors are peptides secreted by the pituitary and hypothalamus as ligands for tyrosine kinase receptors (FGF receptors, FGFRs); FGFs and FGFRs are expressed in many nuclear tissues of epithelial and mesenchymal origin and are involved in the growth, differentiation, and angiogenesis of different organs during embryonic development. They also regulate important physiological behaviors such as proliferation, survival, and migration during human embryonic development, organogenesis, and tissue formation. ${ }^{13}$
In tumors, FGF2 is produced by the tumor cells or the surrounding stromal cells; it has a strong proliferation effect on fibroblasts and can promote the proliferation, migration, and invasion of tumor cells as well as affect endothelial cells to stimulate angiogenesis, which plays a promotional role in tumor growth. Clinical studies have found that FGF2 is overexpressed in malignant tumor tissues (such as prostate cancer, LC, esophageal cancer, and gynecological tumors) and is closely correlated with carcinogenic behaviors, including lymph node metastasis, invasion, migration, and prognosis. ${ }^{14}$ Moreover, increased expression of FGF2 can be detected in a variety of tumor 
tissues and body fluids. Many researchers believe that FGF2 can be considered a tumor marker to predict and provide an early warning for changes in tumors. ${ }^{15}$

Regarding NSCLC, Huang et al suggested that FGF2 might be a target of miR-497-5p, which could reduce the invasion and metastasis of NSCLC by inhibiting FGF2. ${ }^{16}$ Wang et al found that FGF2 is a target gene of miR-646 in NSCLC and that miR-646 might reduce the proliferation and invasion ability of NSCLC by inhibiting FGF2. ${ }^{17}$ In the present study, the FGF2 expression significantly reduced in the A549/DDP cells with overexpression of miR-195-5p upon WB assay and PCR, while the FGF2 expression significantly elevated in the A549 cells with inhibited expression of miR-195-5p. Via the dual-luciferase reporter assay, FGF2 was confirmed to be the direct target gene of miR-195-5p, which could improve the chemosensitivity of cisplatin-resistant LC cells through FGF2 and inhibit migration and invasion.

Subsequently, it was also confirmed by WB assay that the inhibition of chemoresistance of miR-195-5p on FGF2 could be achieved by affecting EMT. ${ }^{18}$ The EMT process is involved in embryogenesis, wound healing, and fibrotic tissue changes and is an important biological process in which malignant tumor cells of epithelial origin acquire the ability to migrate and invade. With the occurrence of EMT in tumor cells, the secretion of intercellular junction protein and epithelial-like marker molecule E-cadherin decreases, while the expressions of mesenchymal cell phenotype N-cadherin, vimentin, and FSP-1 increase. This changes the cell morphology, causing the epithelial tumor cells to lose their polarity and basement membrane connection and adhesion and gradually break away from the binding between the epidermal cells, thus acquiring strong invasive and migratory properties. ${ }^{19}$ EMT leads to the tolerance of tumor cells to cytotoxic drugs and apoptosis regulation and is important in the development of cisplatin resistance in NSCLC. ${ }^{20}$ Currently, extensive literature confirms that EMT and the drug resistance of tumor cells are closely linked and inseparable. ${ }^{21}$ It has been reported that CDDP, doxorubicin, and paclitaxel-resistant cell lines have the characteristics of EMT and a significantly increased cell invasion and metastasis ability compared with their sensitive parental cells. Moreover, cells that develop EMT show stronger drug resistance. ${ }^{22}$ Our results suggested that miR-195-5p could have inhibited the EMT signaling pathway in the A549/DDP cells to improve the cells' resistance to cisplatin. Moreover, it was also confirmed that the overexpression of FGF2 might have promoted EMT in the
A549/DDP cells, while miR-195-5p might have inhibited EMT by reducing the FGF2 expression. We also confirmed that miR-195-5p might have reversed the chemoresistance of the A549/DDP cells by inhibiting FGF2 expression, and miR-195-5p could have reversed the chemoresistance in the A549/DDP cells by inhibiting the expression of the target gene FGF2.

In summary, via cellular and nude mice assays, this study confirmed that there is a differential expression and difference in the cellular functions of miR-195-5p in cisplatin-resistant cells, and it was verified that the direct target gene is FGF2. We also confirmed that miR-195-5p could reverse the chemoresistance in A549/DDP cells by inhibiting the target gene, FGF2, to affect the development of EMT in the resistant cells. We considered that as an oncogene, miR-195-5p might have a potential for drug development and efficacy prediction in the reversal of chemoresistance in NSCLC, with promising practical applications.

\section{Funding}

This study was supported by the National Natural Science Foundation of China (Grant Number: 81772619, 82002551), Clinical Trial Project of Tianjin Medical University (Grant Number: 2017kylc006), and Bethune Charitable Foundation (Grant Number: HZB-2019052818).

\section{Disclosure}

The authors reported no conflicts of interest for this work.

\section{References}

1. Chen W, Zheng R, Baade PD, et al. Cancer statistics in China, 2015. CA Cancer J Clin. 2016;66:115-132. doi:10.3322/caac.21338

2. Jin L, Xu M, Luo XH, Zhu XF. Stephania tetrandra and ginsengcontaining chinese herbal formulation NSENL reverses cisplatin resistance in lung cancer xenografts. Am J Chin Med. 2017;45(2):385-401. doi:10.1142/S0192415X17500240

3. Jayaraj R, Kumarasamy C, Sabarimurugan S, Baxi S. Diagnostic and prognostic value of microRNAs for cancers' strategies and approaches to improve the clinical utility. $J$ Cancer. 2019;10(5):1252-1253. doi: $10.7150 /$ jca. 28581

4. Yu W, Liang X, Li X, et al. MicroRNA-195: a review of its role in cancers. Onco Targets Ther. 2018;11:7109. doi:10.2147/OTT.S183600

5. Hu M, Hu Y, He J, Li B. Prognostic value of basic fibroblast growth factor (bFGF) in lung cancer: a systematic review with meta-analysis. PLoS One. 2016;11(1):e0147374. doi:10.1371/journal.pone.0147374

6. Arrieta O, Lazcano E. Cáncer de pulmón. El peso de la enfermedad y avances en el diagnóstico y tratamiento [Lung cancer. Epidemiology, diagnosis and treatment]. Salud Publica Mex. 2019;61(3):217-218. Spanish. doi:10.21149/10660 
7. Fang L, Niyanhan J, Wenjie L, et al. Research progress on the mechanism of cancer drug resistance. Anti-Tumor Pharm. 2018;8 (3):307-312

8. Jiang T, Dong P, Li L, et al. MicroRNA-200c regulates cisplatin resistance by targeting ZEB2 in human gastric cancer cells. Oncol Rep. 2017;38(1):151-158. doi:10.3892/or.2017.5659

9. Song LY, Ma YT, Wu CF, Wang CJ, Fang WJ, Liu SK. MicroRNA195 activates hepatic stellate cells in vitro by targeting Smad7. Biomed Res Int. 2017;2017. doi:10.1155/2017/1945631

10. Zhang X, Ji R, Liao X, et al. MicroRNA-195 regulates metabolism in failing myocardium via alterations in sirtuin 3 expression and mitochondrial protein acetylation. Circulation. 2018;137(19):2052-2067. doi:10.1161/CIRCULATIONAHA.117.030486

11. Zhang W, Wang Y, Zhu Z, et al. Propofol inhibits proliferation, migration and invasion of gastric cancer cells by up-regulating microRNA-195. Int $J$ Biol Macromol. 2018;120:975-984. doi:10.1016/j.ijbiomac.2018.08.173

12. Zuo W, Zhang W, Xu F, et al. Long non-coding RNA LINC00485 acts as a microRNA-195 sponge to regulate the chemotherapy sensitivity of lung adenocarcinoma cells to cisplatin by regulating CHEK1. Cancer Cell Int. 2019;19(1):1-16. doi:10.1186/s12935-019-0934-7

13. Li Y, Guo XB, Wang JS, Wang HC, Li LP. Function of fibroblast growth factor 2 in gastric cancer occurrence and prognosis. Mol Med Rep. 2020;21(2):575-582. doi:10.3892/mmr.2019.10850

14. Teng Y, Guo B, Mu X, Liu S. KIF26B promotes cell proliferation and migration through the FGF2/ERK signaling pathway in breast cancer. Biomed Pharmacother. 2018;108:766-773. doi:10.1016/j. biopha.2018.09.036

15. Hu N, Kadota M, Liu H, et al. Genomic landscape of somatic alterations in esophageal squamous cell carcinoma and gastric cancer. Cancer Res. 2016;76(7):1714-1723. doi:10.1158/0008-5472.CAN$15-0338$
16. Huang X, Wang L, Liu W, Li F. MicroRNA-497-5p inhibits proliferation and invasion of non-small cell lung cancer by regulating FGF2. Oncol Lett. 2019;17(3):3425-3431. doi:10.3892/ol.2019.9954

17. Wang J, Shu H, Guo S. MiR-646 suppresses proliferation and metastasis of non-small cell lung cancer by repressing FGF2 and CCND2. Cancer Med. 2020;9(12):4360-4370. doi:10.1002/cam4.3062

18. Li F, Zhu T, Yue Y, Zhu X, Wang J, Liang L. Preliminary mechanisms of regulating PD-L1 expression in non-small cell lung cancer during the EMT process. Oncol Rep. 2018;40(2):775-782. doi:10.3892/or.2018.6474

19. Schelch K, Wagner C, Hager S, et al. FGF2 and EGF induce epithelial-mesenchymal transition in malignant pleural mesothelioma cells via a MAPKinase/MMP1 signal. Carcinogenesis. 2018;39(4):534545. doi:10.1093/carcin/bgy018

20. Chen X, Zhao H, Chen C, et al. The HPA/SDC1 axis promotes invasion and metastasis of pancreatic cancer cells by activating EMT via FGF2 upregulation. Oncol Lett. 2020;19(1):211-220. doi:10.3892/ol.2019.11121

21. Kurimoto R, Iwasawa S, Ebata T, et al. Drug resistance originating from a TGF- $\beta$ /FGF-2-driven epithelial-to-mesenchymal transition and its reversion in human lung adenocarcinoma cell lines harboring an EGFR mutation. Int J Oncol. 2016;48(5):1825-1836. doi:10.3892/ ijo.2016.3419

22. Lou Y, Diao L, Cuentas ER, et al. Epithelial-mesenchymal transition is associated with a distinct tumor microenvironment including elevation of inflammatory signals and multiple immune checkpoints in lung adenocarcinoma. Clin Cancer Res. 2016;22(14):3630-3642. doi:10.1158/1078-0432.CCR-15-1434
Pharmacogenomics and Personalized Medicine

\section{Publish your work in this journal}

Pharmacogenomics and Personalized Medicine is an international, peer-reviewed, open access journal characterizing the influence of genotype on pharmacology leading to the development of personalized treatment programs and individualized drug selection for improved safety, efficacy and sustainability. This journal is indexed

\section{Dovepress}

on the American Chemical Society's Chemical Abstracts Service (CAS). The manuscript management system is completely online and includes a very quick and fair peer-review system, which is all easy to use. Visit http://www.dovepress.com/testimonials.php to read real quotes from published authors. 